\title{
Condições de vida de famílias brasileiras: estimativa da insegurança alimentar ${ }^{\star}$
}

\author{
Melissa Luciana de Araújo ${ }^{* *}$ \\ Diana Rodrigues Nascimento ${ }^{\star \star \star}$ \\ Mariana Souza Lopes ${ }^{\star \star \star *}$ \\ Camila Mendes dos Passos ${ }^{\star \star * \star *}$ \\ Aline Cristine Souza Lopes ${ }^{\star \star \star \star \star}$
}

O artigo objetiva estudar a associação entre condições de vida das famílias brasileiras e a estimativa da insegurança alimentar (IA). Para tanto, foi realizado estudo transversal conduzido com dados da Pesquisa de Orçamento Familiar (2008-2009), do IBGE, nas áreas rurais e urbanas. A estimativa da IA foi obtida a partir da pergunta: "das afirmativas a seguir, qual aquela que melhor descreve a quantidade de alimento consumido por sua família?", quando a resposta foi "normalmente não é suficiente" ou "às vezes não é suficiente”. As condições de vida foram analisadas por variáveis econômicas, do domicílio e seu entorno. Modelos de regressão de Poisson com variância robusta, ajustados, foram construídos. Os resultados mostram que, dos 55.406 domicílios investigados, 39,4\% foram classificados em IA, sendo que, na maioria deles, foi identificada dificuldade (93,9\%) para levar a vida até o fim do mês com a renda disponível. Os valores de razão de prevalência, ajustados por renda mensal per capita, região e área, indicam que todos os aspectos econômicos e condições de vida insatisfatórias, bem como os problemas no domicílio e do entorno foram significativamente associados à IA. Conclui-se que é elevada a prevalência da estimativa da IA, sendo associada às condições de vida insatisfatórias das famílias. Portanto, revela-se a necessidade de implementar políticas públicas intersetoriais efetivas visando reduzir as desigualdades.

Palavra-chave: Pesquisa de Orçamento Familiar. Insegurança alimentar. Iniquidade social. Condição de vida.

\footnotetext{
* Os autores agradecem ao professor Dr. Rafael Moreira Claro pelo apoio técnico na elaboração do artigo.

** Universidade Federal de Minas Gerais, Belo Horizonte-MG, Brasil e Universidade Federal de Ouro Preto, Ouro Preto-MG, Brasil (melissaluciana20@gmail.com; https://orcid.org/0000-0002-9932-2135).

*** Universidade Federal de Minas Gerais, Belo Horizonte-MG, Brasil (nutridianarodrigues@gmail.com; https://orcid. org/0000-0002-1095-9835).

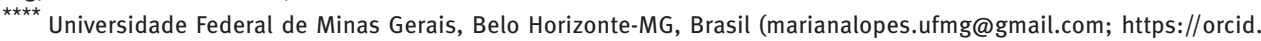
org/0000-0003-3128-7959).

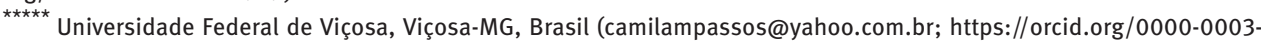
1230-2500).

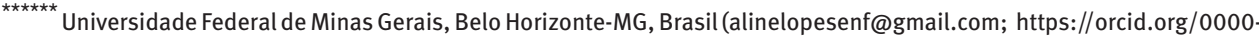
0001-9782-2606).
} 


\section{Introdução}

A abordagem mundial da segurança alimentar e nutricional (SAN) é ampla e dinâmica, em função da história do homem e das sociedades. A temática faz parte de diferentes agendas internacionais e está relacionada às questões de acesso, disponibilidade, direito humano à alimentação adequada, soberania alimentar, biodiversidade, meio ambiente e condicionantes de vida (FAO, 2015; DUTRA et al., 2018).

No Brasil, a SAN é definida como a garantia ao direito humano à alimentação adequada e saudável, à soberania alimentar e ao acesso aos alimentos com qualidade e em quantidade suficiente, sem restringir o acesso a outras necessidades fundamentais e respeitando as diversidades culturais, econômicas e socialmente sustentáveis, tendo práticas alimentares promotoras da saúde como base (BRASIL, 2006, 2014; FAO, 2015). Dessa forma, a SAN assume duas dimensões: a alimentar, relativa ao acesso à qualidade sanitária e biológica e disponibilidade dos alimentos; e a nutricional, que se refere ao estado nutricional, práticas alimentares saudáveis e preservação da cultura alimentar (BRASIL, 2006, 2014; FAO, 2015; ARAUJO et al., 2018). Quando essas dimensões são violadas tem-se, então, a insegurança alimentar (IA).

A IA pode ser vivenciada no domicílio ou individualmente, sendo decorrente de um conjunto de fatores inter-relacionados, com destaque para as desigualdades sociais e condições de vida. Famílias em IA, geralmente, apresentam menor renda per capita, piores ocupações de trabalho, condições de moradia comprometidas, chefe da família do sexo feminino e com menor escolaridade (AIRES et al., 2012; KAC et al., 2012; INTERLENGHI; SALLES-COSTA, 2014; FAO, 2017; ARAÚJO et al., 2018).

A identificação da IA constitui tarefa complexa e desafiadora, especialmente em grandes contingentes populacionais, por requerer o uso de múltiplos indicadores que abranjam suas dimensões sociais, psicológicas, de condições e qualidade de vida (KEPPLE; SEGALLCORREA, 2011; MORAIS, 2014; DUTRA et al., 2014). Sua investigação e compreensão são primordiais para o delineamento de políticas sociais e de saúde que minimizem e detenham seus efeitos deletérios.

Nesse sentido, o monitoramento da IA é preconizado pela Política Nacional de Segurança Alimentar e Nutricional (PNSAN, 2010) (BRASIL, 2015). Resultados deste monitoramento sugerem que, após anos de declínio prolongado, a prevalência de IA no Brasil parece estar em ascendência, corroborando os resultados mundiais (BRASIL, 2015; FAO, 2017). Este aumento da IA pode ser atribuído a diferentes questões, como avanço de governos conservadores e neoliberais, políticas econômicas de austeridade e desaceleração econômica, maior número de conflitos violentos, dentre outras (FAO, 2017).

Este cenário de possível ascensão da IA reforça a necessidade de se compreender melhor como ela se dá no Brasil e possíveis relações com desigualdades das condições de vida. Diante disso, o presente estudo objetivou investigar as condições de vida das famílias brasileiras e a sua relação com a estimativa da IA, segundo dados da Pesquisa de Orçamento Familiar (POF, 2008-2009). 


\section{Metodologia}

\section{Tipo e amostra do estudo}

Trata-se de estudo transversal realizado a partir de dados da POF, obtidos entre maio de 2008 e 2009, pelo Instituto Brasileiro de Geografia e Estatística (IBGE).

A POF objetiva mensurar as estruturas de consumo, gastos, rendimentos e parte da variação de patrimônio das famílias brasileiras, buscando caracterizar suas condições de vida a partir do orçamento doméstico (BRASIL, 2011).

A sua amostra foi composta por domicílios particulares e permanentes do território nacional, abrangendo todas as unidades da federação e áreas urbanas e rurais das cinco regiões (Norte, Nordeste, Sudeste, Sul e Centro-oeste) do país. 0 inquérito utilizou amostra probabilística, com um plano amostral complexo, estratificado e conglomerado, em dois estágios. ${ }^{1}$

Do total de 55.970 domicílios pesquisados pela POF 2008/2009, 564 foram excluídos por não informarem o desfecho de interesse do estudo (situação de IA). Dessa forma, foram investigados 55.406 domicílios, equivalentes a 98,9\% do total analisado na POF.

\section{Organização das variáveis}

Foram utilizados os dados do Suplemento 6 da POF 2008/2009 - "Avaliação das condições de vida" -, que trata da avaliação subjetiva da qualidade de vida das famílias, com base na percepção do responsável pelo domicílio, assim como dados de renda mensal per capita. O Quadro 1 apresenta as variáveis investigadas.

\section{Variável desfecho: estimativa da insegurança alimentar}

A variável desfecho investigada, estimativa da IA do domicílio, foi obtida a partir da questão 4 do Suplemento (Quadro 1): “das afirmativas a seguir, qual aquela que melhor descreve a quantidade de alimento consumido por sua família? ", que apresentava as seguintes opções de resposta: “normalmente não é suficiente”; “às vezes não é suficiente”; e "é sempre suficiente". Foi classificado em IA o domicílio cuja resposta foi "normalmente não é suficiente” ou “às vezes não é suficiente” e, em situação de segurança alimentar (SA), aquele com a resposta "é sempre suficiente". A escolha desta variável para avaliação de IA deveu-se à ausência de dados oriundos da Escala Brasileira de Insegurança Alimentar (Ebia) na POF, sendo, portanto, dentre as disponíveis, a variável que mais se aproximava de uma estimativa da IA.

\footnotetext{
${ }^{1}$ Mais detalhes sobre o procedimento amostral e as condutas empregadas para coleta de dados podem ser obtidos em publicação específica (BRASIL, 2011).
} 


\section{QUADRO 1}

Questões utilizadas para caracterizar aspectos econômicos, condições de vida e problemas no domicílio conforme a situação de segurança alimentar e nutricional dos domicílios Brasil - 2008/2009

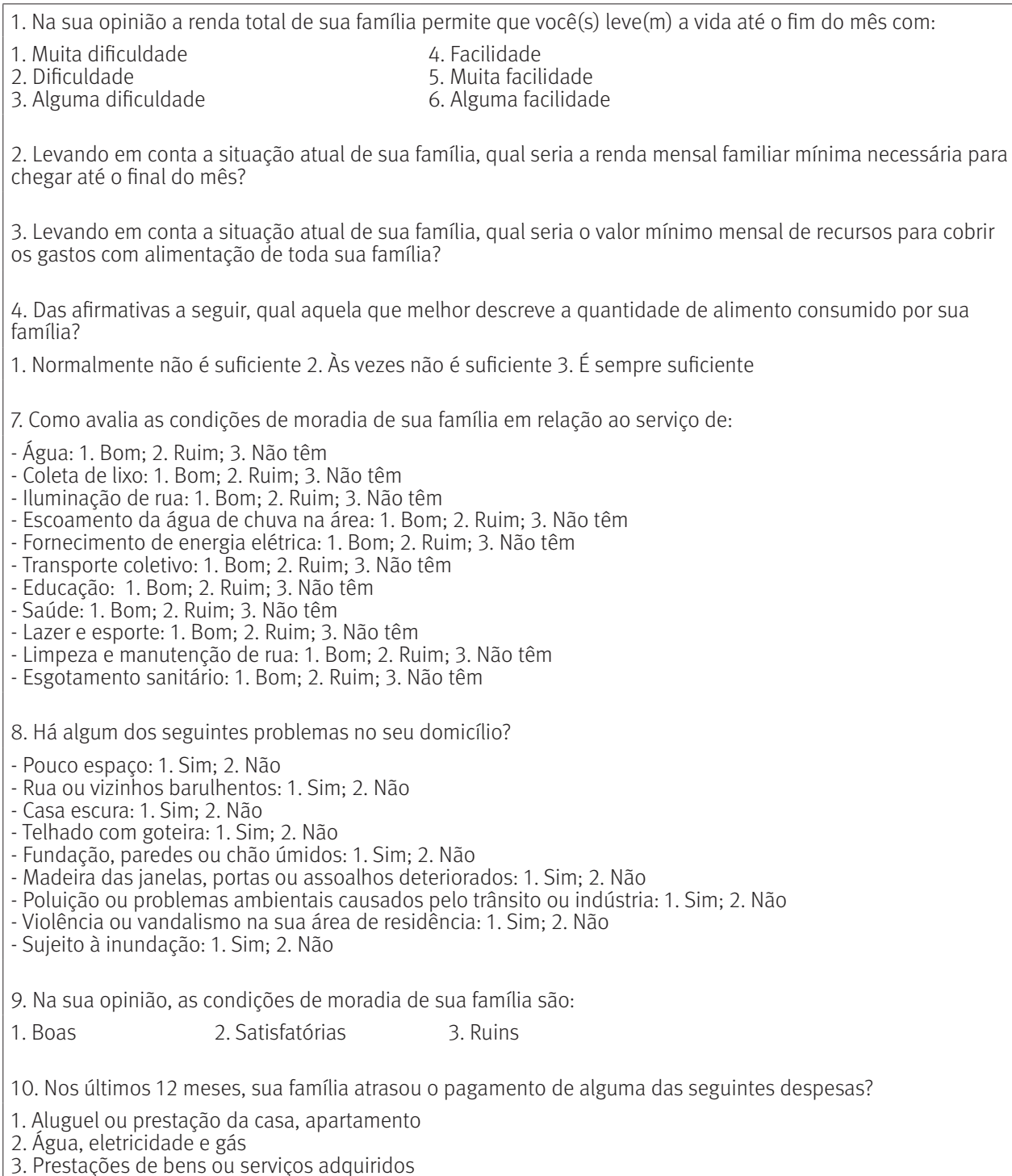

Fonte: IBGE. Pesquisa de Orçamentos Familiares (POF) 2008-2009. Suplemento 6: Avaliação das Condições de Vida.

\section{Variáveis explicativas}

As condições de vida das famílias brasileiras foram analisadas segundo os aspectos econômicos (questões de 1 a 3 e 10), autoavaliação das condições de vida (questões 7 e 9) e condições do domicílio e do entorno (questão 8) (Quadro 1). 
Os aspectos econômicos abarcaram as seguintes variáveis:

- dificuldade de sobrevivência com a renda total familiar até o fim do mês (agrupada em: $\operatorname{sim}=$ muita dificuldade/dificuldade/alguma dificuldade; e não = alguma facilidade/facilidade/muita facilidade);

- renda mensal familiar mínima desejada de até dois salários mínimos para chegar até o fim do mês (sim, não), obtida pela questão “levando em conta a situação atual da sua família, qual seria a renda familiar mínima necessária para chegar até o final do mês?";

- mínimo mensal de recursos para cobrir gastos com a alimentação de até dois salários mínimos (sim, não), obtida pela questão “levando em conta a situação atual de sua família, qual seria o valor mínimo mensal de recursos para cobrir os gastos com alimentação de toda sua família?";

- atraso nos pagamentos de despesas essenciais: aluguel ou prestação do imóvel (sim, não); pagamento de água, luz e gás (sim, não); e pagamento de bens ou serviços (sim, não).

Para as condições de vida autoavaliadas foram analisadas as variáveis: condições de moradia (boas, satisfatórias, ruins); serviço de água (bom, ruim, não tem); serviço de coleta de lixo (bom, ruim, não tem); iluminação da rua (boa, ruim, não tem); serviço de limpeza e manutenção de rua (bom, ruim, não tem); escoamento da água de chuva (bom, ruim, não tem); serviço de rede elétrica (bom, ruim, não tem); serviço de transporte coletivo (bom, ruim, não tem); serviço de educação (bom, ruim, não tem); serviço de saúde (bom, ruim, não tem); acesso a atividade de lazer e esportes (bom, ruim, não tem); e serviço de esgoto sanitário (bom, ruim, não tem). As respostas ruim e não tem, para as condições de vida, foram consideradas como "insatisfatórias".

Para as condições dos domicílios e do entorno foram analisadas as variáveis: pouco espaço (sim, não); rua e vizinhos barulhentos (sim, não); casa escura (sim, não); telhado com goteira (sim, não); fundação, paredes ou chão úmidos (sim, não); madeira das janelas, portas ou assoalhos deteriorados (sim, não); poluição ou problemas ambientais devido a trânsito ou indústria (sim, não); violência ou vandalismo na área de residência (sim, não); e sujeito à inundação (sim, não). As respostas afirmativas para as condições dos domicílios foram consideradas como "insatisfatórias".

Variáveis de ajuste

Utilizaram-se, como variáveis de ajuste, a renda mensal per capita, a região do país (Norte, Nordeste, Sudeste, Sul e Centro-oeste) e se residia em área urbana ou rural.

\section{Análises de dados}

Análises de associação entre as varáveis explicativas e a estimativa de IA do domicílio (desfecho) foram realizadas por modelos de regressão de Poisson com variância robusta, 
univariados e multivariados, que geraram razões de prevalências não ajustadas e ajustadas e seus respectivos intervalos de $95 \%$ de confiança. Foram obtidos os seguintes modelos: Modelo 1, ajustado por renda mensal per capita; e Modelo 2, ajustado por renda mensal per capita, região e área do país.

Para organização e análises estatísticas utilizou-se o software Stata, versão 14.0, considerando os fatores de ponderação e os aspectos da amostragem complexa da POF 2008-2009.

\section{Resultados}

A prevalência de domicílios com IA foi de 39,4\% ( $n=21.826)$, sendo que na maioria deles $(93,9 \%)$ havia dificuldade de sobrevivência até o fim do mês com a renda disponível. Quase a totalidade de domicílios investigados considerou que dois salários mínimos seria o valor mínimo mensal necessário para as despesas mensais e que o valor mínimo mensal para os gastos com a alimentação deveria variar até dois salários mínimos (99,5\% e 99,4\%, respectivamente) (Tabela 1$)$.

O atraso no pagamento de despesas, como aluguel ou prestação de imóvel, água, luz, gás e bens ou serviços nos últimos 12 meses, também registrou maior proporção nas famílias em situação de IA. Menor percentual de atraso foi verificado no pagamento do aluguel ou da prestação do imóvel $(6,2 \%)$, seguido de bens ou serviços $(37,3 \%)$ e do pagamento de água, luz e gás (49,1\%) (Tabela 1).

A maior parte das famílias brasileiras em situação de IA avaliou as condições de moradia como insatisfatórias (65,9\%). Verificou-se, ainda, que mais da metade dessas famílias relatou insatisfação com serviço de limpeza e manutenção de ruas $(65,7 \%)$, escoamento de água da chuva $(51,8 \%)$, serviço de transporte coletivo $(64,8 \%)$, serviço de saúde $(65,8 \%)$, serviço de esgotamento sanitário $(70,4 \%)$ e acesso a atividades de lazer e esportes $(79,1 \%)$. Prevalências menores de insatisfação foram identificadas com os serviços de água (38,4\%), coleta de lixo (38,6\%), iluminação (46,1\%), educação $(37,7 \%)$ e rede elétrica (18,7\%) (Tabela 1$)$.

As famílias brasileiras em situação de IA avaliaram as condições de seu domicílio como piores, quando comparadas àquelas em situação de segurança alimentar, exceto quando questionadas sobre a poluição ou problemas ambientais devido a trânsito ou indústria $(I A=16,6 \%$ vs. $S A=17,0 \%)$. Os problemas no domicílio mais relatados foram pouco espaço (51,9\%), telhado com goteira $(49,8 \%)$, madeira das janelas, portas ou assoalhos deteriorados (43,7\%), fundação, parede ou chão úmidos (42,8\%) e violência e vandalismo na área da residência (32,9\%) (Tabela 2$)$. 
TABELA 1

Domicílios, por situação de segurança alimentar, segundo aspectos econômicos e condições de vida autoavaliadas Brasil - 2008/2009

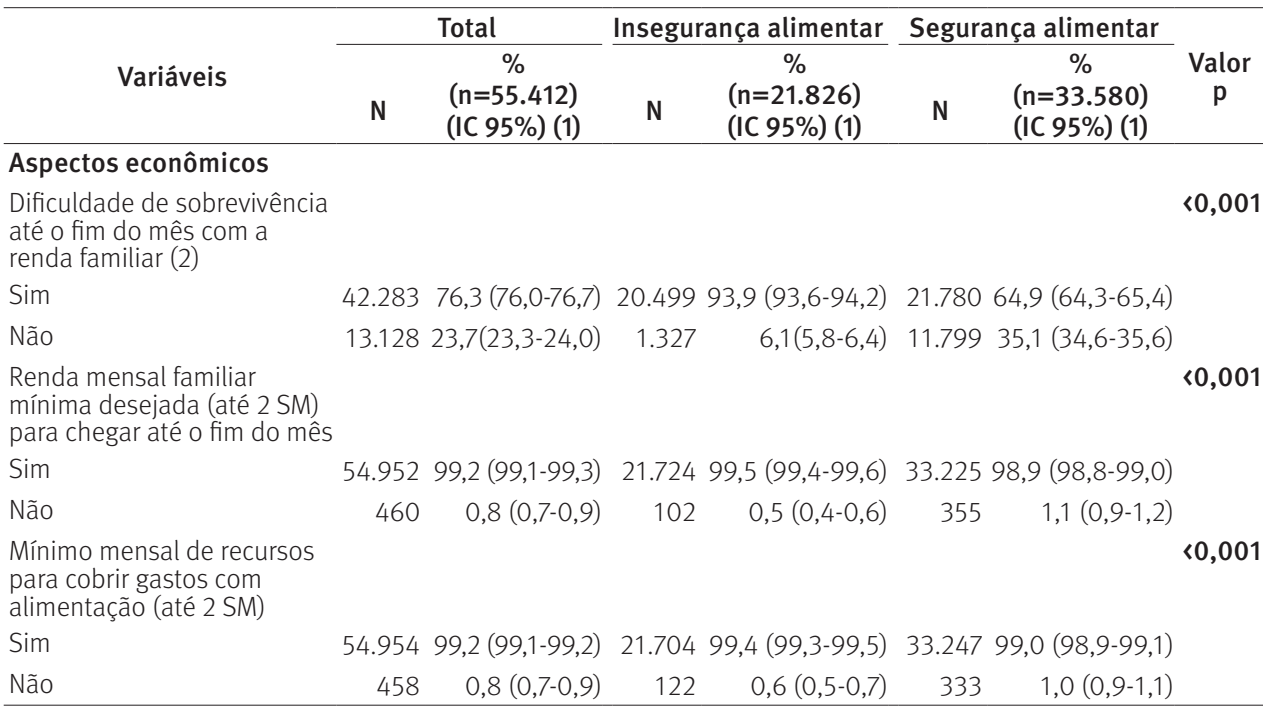

\section{Atraso no pagamento}

Aluguel ou prestação do imóvel (3)

\begin{tabular}{|c|c|c|c|c|c|c|c|}
\hline $\operatorname{Sim}$ & 2.865 & $5,2(5,0-5,4)$ & 1.357 & $6,2(5,9-6,5)$ & 1.508 & $4,5(4,3-4,7)$ & \\
\hline Não & 52.533 & $94,8(94,6-95,04)$ & 20.464 & $93,8(93,4-94,1)$ & 32.066 & $95,5(95,3-95,7)$ & \\
\hline Água, luz e gás (4) & & & & & & & $<0,001$ \\
\hline Sim & 20.680 & $37,3(36,9-37,7)$ & 10.715 & $49,1(48,4-49,8)$ & 9.963 & $29,7(29,2-30,2)$ & \\
\hline Não & 34.722 & $62,8(62,3-63,1)$ & 11.108 & $50,9(50,2-51,6)$ & 23.613 & $70,3(69,8-70,8)$ & \\
\hline Bens ou serviços (5) & & & & & & & $<0,001$ \\
\hline Sim & 15.960 & $28,8(28,4-29,2)$ & 8.142 & $37,3(36,7-37,9)$ & 7.816 & $23,3(22,8-23,7)$ & \\
\hline Não & 39.440 & $71,2(70,8-71,6)$ & 13.681 & $62,7(62,0-63,3)$ & 25.758 & $76,7(76,3-77,2)$ & \\
\hline \multicolumn{8}{|l|}{$\begin{array}{l}\text { Condições de vida } \\
\text { insatisfatórias }\end{array}$} \\
\hline Moradia (6) & & & & & & & $<0,001$ \\
\hline Sim & 27.875 & $50,3(49,9-50,7)$ & 14.383 & $65,9(65,3-66,5)$ & 13.490 & $40,2(39,6-40,7)$ & \\
\hline Não & 27.529 & $49,7(49,3-50,1)$ & 7.442 & $34,1(33,5-34,7)$ & 20.086 & $59,8(59,3-60,3)$ & \\
\hline Serviço de água (7) & & & & & & & $<0,001$ \\
\hline Sim & 16.926 & $30,6(30,1-30,9)$ & 8.382 & $38,4(37,8-39,0)$ & 8.542 & $25,4(25,0-25,9)$ & \\
\hline Não & 38.484 & $69,4(69,1-69,8)$ & 13.444 & $61,6(60,9-62,2)$ & 25.038 & $74,6(74,0-75,0)$ & \\
\hline Serviço de esgoto sanitário (8) & & & & & & & $<0,001$ \\
\hline $\operatorname{Sim}$ & 21.674 & $28,8(28,4-29,2)$ & 15.374 & $70,4(69,8-71,1)$ & 18.344 & $54,6(54,1-55,2)$ & \\
\hline Não & 33.722 & $60,9(60,5-61,3)$ & 6.446 & $29,6(28,9-30,1)$ & 15.228 & $45,4(44,8-45,9)$ & \\
\hline Serviço de coleta de lixo (7) & & & & & & & $<0,001$ \\
\hline $\operatorname{Sim}$ & 17.588 & $31,7(31,4-32,1)$ & 8.424 & $38,6(37,9-39,2)$ & 9.163 & $27,3(26,8-27,8)$ & \\
\hline Não & 37.822 & $68,3(67,9-68,6)$ & 13.402 & $61,4(60,8-62,0)$ & 24.417 & $72,7(72,2-73,2)$ & \\
\hline Iluminação da rua (7) & & & & & & & $<0,001$ \\
\hline $\operatorname{Sim}$ & 22.813 & $41,2(40,8-41,6)$ & 10.052 & $46,1(45,4-46,7)$ & 12.760 & $38,0(37,5-38,5)$ & \\
\hline Não & 32.597 & $58,8(58,4-59,2)$ & 11.774 & $53,9(53,3-54,6)$ & 20.820 & $62,0(61,5-62,5)$ & \\
\hline
\end{tabular}


(continuação)

\begin{tabular}{|c|c|c|c|c|c|c|c|}
\hline \multirow[b]{2}{*}{ Variáveis } & \multicolumn{2}{|r|}{ Total } & \multicolumn{2}{|c|}{ Insegurança alimentar } & \multicolumn{2}{|c|}{ Segurança alimentar } & \multirow[b]{2}{*}{$\begin{array}{c}\text { Valor } \\
\mathrm{p}\end{array}$} \\
\hline & $\mathrm{N}$ & $\begin{array}{c}\% \\
(n=55.412) \\
(I C 95 \%)(1)\end{array}$ & $\mathbf{N}$ & $\begin{array}{c}\% \\
(n=21.826) \\
(I C 95 \%)(1)\end{array}$ & $\mathrm{N}$ & $\begin{array}{c}\% \\
(n=33.580) \\
(I C 95 \%)(1)\end{array}$ & \\
\hline $\begin{array}{l}\text { Serviço de limpeza e } \\
\text { manutenção de rua (9) }\end{array}$ & & & & & & & $<0,001$ \\
\hline Sim & 32.580 & $58,8(58,4-59,2)$ & 14.348 & $65,7(65,1-66,4)$ & 18.230 & $54,3(53,8-54,8)$ & \\
\hline Não & 22.819 & $41,2(40,8-41,6)$ & 7.465 & $34,3(33,6-34,9)$ & 15.343 & $45,7(45,2-46,2)$ & \\
\hline $\begin{array}{l}\text { Escoamento da água de } \\
\text { chuva (10) }\end{array}$ & & & & & & & $<0,001$ \\
\hline Sim & 24.753 & $44,7(44,3-45,1)$ & 11.312 & $51,8(51,2-52,5)$ & 13.438 & $40,0(39,5-40,5)$ & \\
\hline Não & 30.653 & $55,3(54,9-55,7)$ & 10.513 & $48,2(47,5-48,8)$ & 20.139 & $60,0(59,4-60,5)$ & \\
\hline Serviço de rede elétrica (11) & & & & & & & $<0,001$ \\
\hline Sim & 7.619 & $13,8(13,4-14,0)$ & 4.084 & $18,7(18,2-19,2)$ & 3.535 & $10,6(10,2-10,8)$ & \\
\hline Não & 47.790 & $86,2(86,0-86,5$ & 17.741 & $81,3(80,8-81,8)$ & 30.045 & $89,4(89,1-89,8$ & \\
\hline $\begin{array}{l}\text { Serviço de transporte } \\
\text { coletivo (12) }\end{array}$ & & & & & & & $<0,001$ \\
\hline Sim & 22.981 & $41,5(41,1-41,9)$ & 14.149 & $64,8(64,2-65,5)$ & 18.254 & $54,4(53,9-54,9)$ & \\
\hline Não & 32.406 & $58,5(58,1-58,9)$ & 7.672 & $35,2(34,5-35,8)$ & 15.308 & $45,6(45,1-46,1)$ & \\
\hline Serviço de educação (13) & & & & & & & $<0,001$ \\
\hline Sim & 18.458 & $33,3(33,0-33,7)$ & 8.220 & $37,7(37,0-38,3)$ & 10.236 & $30,5(30,0-31,0)$ & \\
\hline Não & 36.918 & $66,7(66,3-67,1)$ & 13.597 & $62,3(61,7-63,0)$ & 23.319 & $69,5(69,0-70,0)$ & \\
\hline Serviço de saúde (14) & & & & & & & $<0,001$ \\
\hline Sim & 22.801 & $41,2(40,7-41,6)$ & 14.354 & $65,8(65,1-66,4)$ & 18.235 & $54,3(53,8-54,8)$ & \\
\hline Não & 32.591 & $58,8(58,4-59,2)$ & 7.466 & $34,2(33,6-34,8)$ & 15.333 & $45,7(45,1-46,2)$ & \\
\hline $\begin{array}{l}\text { Acesso a atividade de lazer e } \\
\text { esportes (15) }\end{array}$ & & & & & & & $<0,001$ \\
\hline Sim & 40.130 & $72,4(72,1-72,8)$ & 17.262 & $79,1(78,6-79,6)$ & 22.864 & $68,1(67,6-68,6)$ & \\
\hline Não & 15.254 & $27,6(27,2-27,9)$ & 4.550 & $20,4(19,9-20,9)$ & 10.704 & $31,9(31,4-32,4)$ & \\
\hline
\end{tabular}

Fonte: IBGE. Pesquisa de Orçamentos Familiares (POF) 2008-2009. Suplemento 6: Avaliação das Condições de Vida. Elaboração dos autores.

(1) Intervalo de Confiança de 95\%. (2) Um não respondeu. (3) 14 não responderam. (4) 10 não responderam. (5) 12 não responderam. (6) 8 não responderam. (7) 2 não responderam. (8) 16 não responderam. (9) 13 não responderam. (10) 6 não responderam. (11) 3 não responderam. (12) 25 não responderam. (13) 36 não responderam. (14) 20 não responderam. (15) 28 não responderam.

Nota: $O$ salário mínimo vigente na época era de $\mathrm{R} \$ 415,00$.

Em relação aos aspectos econômicos, após o ajustamento, todos foram associados à estimativa de IA, sendo que os maiores valores de razão de prevalência foram encontrados para os domicílios expostos a dificuldade de sobrevivência com a renda familiar até o fim do mês ( $R P=3,81$; IC95\% 3,49 - 4,16) e renda mensal familiar mínima desejada de até dois salários mínimos para chegar até o fim do mês (RP = 2,00; IC95\% 1,54 - 2,60) (Tabela 3).

Todas as condições de vida avaliadas como insatisfatórias também se associaram à estimativa de IA, após ajustamento, sendo as maiores magnitudes da razão de prevalência encontradas para condições de moradia ( $R P=1,61$; IC95\% 1,56 - 1,67), serviço de saúde $(R P=1,27 ; I C 95 \% 1,22-1,31)$, acesso a atividade de lazer e esportes ( $R P=1,26 ;$ IC95\% 1,21 - 1,32) e serviço de educação ( $R P=1,23$; IC95\% 1,19 - 1,27). Problemas no domicílio e do entorno também foram associados à estimativa de IA (Tabela 3). 
TABELA 2

Domicílios, por situação de segurança alimentar, segundo problemas no domicílio e entrono Brasil - 2008/2009

\begin{tabular}{|c|c|c|c|c|c|c|c|}
\hline \multirow[b]{2}{*}{ Variáveis } & \multicolumn{2}{|r|}{ Total } & \multicolumn{2}{|c|}{ Insegurança alimentar } & \multicolumn{2}{|c|}{ Segurança alimentar } & \multirow[b]{2}{*}{$\begin{array}{l}\text { Valor } \\
\text { P }\end{array}$} \\
\hline & $\mathrm{N}$ & $\begin{array}{c}\% \\
(n=55.412) \\
(I C 95 \%)(1)\end{array}$ & $\mathrm{N}$ & $\begin{array}{c}\% \\
(n=21.826) \\
(I C 95 \%)(1)\end{array}$ & $\mathrm{N}$ & $\begin{array}{c}\% \\
(n=33.580) \\
(I C 95 \%)(1)\end{array}$ & \\
\hline Pouco espaço (2) & & & & & & & $<0,001$ \\
\hline $\operatorname{Sim}$ & 21.560 & $38,9(38,5-39,3)$ & 11.323 & $51,9(51,2-52,5)$ & 10.236 & $30,5(30,0-31,0)$ & \\
\hline Não & 33.846 & $61,1(60,7-61,5)$ & 10.502 & $48,1(47,5-48,8)$ & 23.342 & $69,5(69,0-70,0)$ & \\
\hline $\begin{array}{l}\text { Rua e vizinhos } \\
\text { barulhentos (2) }\end{array}$ & & & & & & & $<0,001$ \\
\hline Sim & 13.526 & $24,4(24,1-24,8)$ & 5.698 & $26,1(25,5-26,7)$ & 7.827 & $23,3(22,9-23,8)$ & \\
\hline Não & 41.880 & $75,6(75,2-75,9)$ & 16.127 & $73,9(73,3-74,5)$ & 25.751 & $76,7(76,2-77,1)$ & \\
\hline Casa escura (3) & & & & & & & $<0,001$ \\
\hline Sim & 10.916 & $19,7(19,4-20,0)$ & 6.089 & $27,9(27,3-28,5)$ & 4.827 & $14,4(14,0-14,8)$ & \\
\hline Não & 44.488 & $80,3(79,9-80,6)$ & 15.735 & $72,1(71,5-72,7)$ & 28.750 & $85,6(85,2-86,0)$ & \\
\hline Telhado com goteira (4) & & & & & & & $<0,001$ \\
\hline $\operatorname{Sim}$ & 19.799 & $35,7(35,3-36,1)$ & 10.856 & $49,8(49,1-50,4)$ & 8.941 & $26,6(26,2-27,1)$ & \\
\hline Não & 35.606 & $64,3(63,9-64,7)$ & 10.967 & $50,2(49,6-50,9)$ & 24.638 & $73,4(72,9-73,8)$ & \\
\hline $\begin{array}{l}\text { Fundação, paredes ou } \\
\text { chão úmidos (3) }\end{array}$ & & & & & & & $<0,001$ \\
\hline $\operatorname{Sim}$ & 17.627 & $31,8(31,4-32,2)$ & 9.343 & $42,8(42,2-43,5)$ & 8.282 & $24,7(24,2-25,1)$ & \\
\hline Não & 37.777 & $68,2(67,8-68,6)$ & 12.481 & $57,2(56,5-57,8)$ & 25.295 & $75,3(74,9-75,8)$ & \\
\hline $\begin{array}{l}\text { Madeira das janelas, } \\
\text { portas ou assoalhos } \\
\text { deteriorados (5) }\end{array}$ & & & & & & & $<0,001$ \\
\hline $\operatorname{Sim}$ & 16.850 & $30,4(30,0-30,8)$ & 9.531 & $43,7(43,0-44,3)$ & 7.316 & $21,8(21,3-22,2)$ & \\
\hline Não & 38.550 & $69,6(69,2-70,0)$ & 12.290 & $56,3(55,7-57,0)$ & 26.260 & $78,2(77,8-78,6)$ & \\
\hline $\begin{array}{l}\text { Poluição ou problemas } \\
\text { ambientais } \\
\text { devido a trânsito ou } \\
\text { indústria (6) }\end{array}$ & & & & & & & 0,288 \\
\hline $\operatorname{Sim}$ & 9.337 & $16,8(16,5-17,2)$ & 3.631 & $16,6(16,1-17,1)$ & 5.703 & $17,0(16,6-17,4)$ & \\
\hline Não & 46.065 & $83,2(82,8-83,5)$ & 18.191 & $83,4(82,9-83,8)$ & 27.874 & $83,0(82,6-83,4)$ & \\
\hline $\begin{array}{l}\text { Violência ou vandalismo } \\
\text { na área de } \\
\text { residência (7) }\end{array}$ & & & & & & & $<0,001$ \\
\hline $\operatorname{Sim}$ & 16.482 & $29,7(29,4-30,1)$ & 7.184 & $32,9(32,3-33,5)$ & 9.297 & $27,7(27,2-28,2)$ & \\
\hline Não & 38.919 & $70,3(69,9-70,6)$ & 14.639 & $67,1(66,4-67,7)$ & 24.278 & $72,3(71,8-72,8)$ & \\
\hline Sujeito à inundação (7) & & & & & & & $<0,001$ \\
\hline $\operatorname{Sim}$ & 5.392 & $9,7(9,5-10,0)$ & 2.777 & $12,7(12,0-13,2)$ & 2.614 & $7,8(7,5-8,1)$ & \\
\hline Não & 50.009 & $90,3(90,0-90,5)$ & 19.046 & $87,3(86,8-87,7)$ & 30.961 & $92,2(91,9-92,5)$ & \\
\hline
\end{tabular}

Fonte: IBGE. Pesquisa de Orçamentos Familiares (POF) 2008-2009. Suplemento 6: Avaliação das Condições de Vida. Elaboração dos autores.

(1) Intervalo de confiança de $95 \%$. (2) 6 não responderam. (3) 8 não responderam. (4) 7 não responderam. (5) 12 não responderam. (6) 10 não responderam. (7) 11 não responderam. 
TABELA 3

Razão de prevalência da estimativa de insegurança alimentar (IA), segundo aspectos econômicos, condições de vida autoavaliadas e problemas no domicílio e entorno, ajustados por renda familiar per capita, região e área Brasil - 2008/2009

\begin{tabular}{|c|c|c|c|c|c|c|}
\hline Variáveis & RP bruta & IC $95 \%$ & $\begin{array}{c}\mathrm{RP} \\
\text { ajustado } \\
\text { modelo } \\
1^{\star}\end{array}$ & IC $95 \%$ & $\begin{array}{c}\mathrm{RP} \\
\text { ajustado } \\
\text { modelo } \\
2^{\star \star}\end{array}$ & IC $95 \%$ \\
\hline \multicolumn{7}{|l|}{ Aspectos econômicos } \\
\hline $\begin{array}{l}\text { Dificuldade de sobrevivência até o fim do } \\
\text { mês com a renda familiar }\end{array}$ & $5,36^{\star \star \star}$ & $4,92-5,85$ & $3,79^{\star \star \star}$ & $3,47-4,13$ & $3,81^{\star \star \star}$ & $3,49-4,16$ \\
\hline $\begin{array}{l}\text { Renda mensal familiar mínima desejada } \\
\text { (até } 2 \text { SM) para chegar até o fim do mês }\end{array}$ & $2,34^{\star \star \star}$ & $1,76-3,13$ & 1,98 & $1,52-2,58$ & 2,00 & $1,54-2,60$ \\
\hline $\begin{array}{l}\text { Mínimo mensal de recursos para cobrir } \\
\text { gastos com alimentação (até } 2 \text { SM) }\end{array}$ & $1,70^{\star \star \star}$ & $1,31-2,21$ & 1,39 & $1,08-1,78$ & $1,40^{\star \star \star \star}$ & $1,10-1,80$ \\
\hline \multicolumn{7}{|l|}{ Atraso do pagamento } \\
\hline Aluguel ou prestação do imóvel & $1,24^{\star \star \star}$ & $1,16-1,33$ & $1,18^{\star \star \star}$ & $1,11-1,26$ & $1,20^{\star \star \star}$ & $1,13-1,28$ \\
\hline Pagamento de água, luz e gás & $1,72^{\star \star \star}$ & $1,66-1,77$ & $1,39^{\star \star \star}$ & $1,34-1,43$ & $1,37^{\star \star \star}$ & $1,33-1,42$ \\
\hline Pagamento de bens ou serviços & $1,53^{\star \star \star}$ & $1,48-1,58$ & $1,31^{\star \star \star}$ & $1,27-1,35$ & $1,30^{\star \star \star}$ & $1,26-1,34$ \\
\hline \multicolumn{7}{|l|}{ Condições de vida insatisfatórias } \\
\hline Moradia & $2,05^{\star \star \star}$ & $1,97-2,12$ & $1,62^{\star \star \star}$ & $1,56-1,68$ & $1,61^{\star \star \star}$ & $1,56-1,67$ \\
\hline Serviço de água & $1,52^{\star \star \star}$ & $1,47-1,57$ & $1,18^{\star \star \star}$ & $1,14-1,22$ & $1,16^{\star \star \star}$ & $1,12-1,20$ \\
\hline Serviço de esgoto sanitário & $1,64^{\star \star \star}$ & $1,58-1,70$ & $1,20^{\star \star \star}$ & $1,16-1,25$ & $1,19^{\star \star \star}$ & $1,14-1,24$ \\
\hline Serviço de coleta de lixo & $1,50^{\star \star \star}$ & $1,45-1,55$ & $1,10^{\star \star \star}$ & $1,07-1,14$ & $1,12^{\star \star \star}$ & $1,09-1,17$ \\
\hline Iluminação da rua & $1,29^{\star \star \star}$ & $1,25-1,34$ & $1,08^{\star \star \star}$ & $1,05-1,11$ & $1,09^{\star \star \star}$ & $1,06-1,13$ \\
\hline Limpeza e manutenção de rua & $1,41^{\star \star \star}$ & $1,37-1,47$ & $1,15^{\star \star \star}$ & $1,11-1,19$ & $1,15^{\star \star \star}$ & $1,11-1,19$ \\
\hline Escoamento da água de chuva & $1,42^{\star \star \star}$ & $1,38-1,47$ & $1,16^{\star \star \star}$ & $1,12-1,20$ & $1,15^{\star \star \star}$ & $1,11-1,18$ \\
\hline Serviço de rede elétrica & $1,51^{\star \star \star}$ & $1,45-1,57$ & $1,23^{\star \star \star}$ & $1,19-1,28$ & $1,22^{\star \star \star}$ & $1,18-1,27$ \\
\hline Serviço de transporte coletivo & $1,34^{\star \star \star}$ & $1,30-1,39$ & $1,12^{\star \star \star}$ & $1,08-1,16$ & $1,10^{\star \star \star}$ & $1,06-1,14$ \\
\hline Serviço de educação & $1,26^{\star \star \star}$ & $1,22-1,31$ & $1,24^{\star \star \star}$ & $1,20-1,28$ & $1,23^{\star \star \star}$ & $1,19-1,27$ \\
\hline Serviço de saúde & $1,42^{\star \star \star}$ & $1,37-1,39$ & $1,29^{\star \star \star}$ & $1,25-1,29$ & $1,27^{\star \star \star}$ & $1,22-1,31$ \\
\hline Acesso a atividade de lazer e esportes & $1,55^{\star \star \star}$ & $1,48-1,62$ & $1,28^{\star \star \star}$ & $1,23-1,33$ & $1,26^{\star \star \star}$ & $1,21-1,32$ \\
\hline \multicolumn{7}{|l|}{ Problemas no domicílio e entorno } \\
\hline Pouco espaço & $1,76^{\star \star \star}$ & $1,70-1,82$ & $1,32^{\star \star \star}$ & $1,28-1,31$ & $1,32^{\star \star \star}$ & $1,27-1,36$ \\
\hline Rua e vizinhos barulhentos & $1,04^{\star \star \star \star}$ & $1,00-1,08$ & $1,08^{\star \star \star}$ & $1,04-1,11$ & $1,06^{\star \star \star \star}$ & $1,03-1,10$ \\
\hline Casa escura & $1,67^{\star \star \star}$ & $1,61-1,72$ & $1,35^{\star \star \star}$ & $1,31-1,40$ & $1,35^{\star \star \star}$ & $1,31-1,39$ \\
\hline Telhado com goteira & $1,84^{\star \star \star}$ & $1,83-1,95$ & $1,40^{\star \star \star}$ & $1,36-1,45$ & $1,38^{\star \star \star}$ & $1,34-1,43$ \\
\hline Fundação, paredes ou chão úmidos & $1,63^{\star \star \star}$ & $1,58-1,69$ & $1,32^{\star \star \star}$ & $1,28-1,36$ & $1,30^{\star \star \star}$ & $1,26-1,34$ \\
\hline $\begin{array}{l}\text { Madeira de janelas/portas/ assoalhos } \\
\text { deteriorados }\end{array}$ & $1,88^{\star \star \star}$ & $1,81-1,93$ & $1,38^{\star \star \star}$ & $1,34-1,43$ & $1,37^{\star \star \star}$ & $1,33-1,41$ \\
\hline Violência ou vandalismo na área & $1,19^{\star \star \star}$ & $1,15-1,24$ & $1,19^{\star \star \star}$ & $1,15-1,24$ & $1,17^{\star \star \star}$ & $1,14-1,22$ \\
\hline Sujeito à inundação & $1,35^{\star \star \star}$ & $1,28-1,42$ & $1,20^{\star \star \star}$ & $1,15-1,25$ & $1,19^{\star \star \star}$ & $1,14-1,24$ \\
\hline
\end{tabular}

Fonte: IBGE. Pesquisa de Orçamentos Familiares (POF) 2008-2009. Suplemento 6: Avaliação das Condições de Vida. Elaboração dos autores.

RP: razão de prevalência. IC 95\%: intervalo 95\% de confiança. SM: salário mínimo.

* Ajustada pela variável de renda mensal per capita.

**Ajustada pelas variáveis de renda mensal per capita, região do país e área de residência (urbano ou rural).

*** Variáveis significativas ( $p<0,001)$ no modelo de regressão de Poisson ajustado pelo fator de expansão.

$\star \star \star *$ Variáveis significativas $(p<0,05)$ no modelo de regressão de Poisson ajustado pelo fator de expansão. 


\section{Discussão}

Aproximadamente $40 \%$ das famílias brasileiras, em 2008-2009, estavam em situação de IA, segundo estimativa obtida a partir da POF. Estas famílias, quando comparadas àquelas em $\mathrm{SA}$, revelaram viver em cenários mais desfavoráveis, enfrentando dificuldades para suprir suas necessidades com a renda disponível e diversos problemas estruturais nos domicílios e no entorno, além de acesso inadequado a serviços salutares, como saneamento básico, transporte, saúde e educação.

A prevalência de estimativa de IA identificada neste estudo foi elevada e superior a outros países da América Latina (BRASIL, 2015), como México (URQUÍA-FERNANDES, 2014) e Colômbia (CASTANÕ; ISAZA, 2013). A IA tem sido investigada em várias populações, utilizando diferentes métodos de mensuração, o que pode explicar, em parte, estas diferenças (URQUÍA-FERNANDES, 2014;CASTANÕ; ISAZA, 2013; PÉREZ-ESCAMILLA et al., 2004).

A maior parte das famílias investigadas em situação de IA apresentava dificuldades para arcar com suas despesas até o final do mês e considerava dois salários mínimos a renda familiar mínima para suprir as despesas mensais e cobrir os gastos com alimentação. A renda familiar é um dos indicadores com maior impacto sobre a IA, tanto em áreas urbanas quanto rurais. Estudo realizado na região metropolitana do Rio de Janeiro mostrou que famílias com renda per capita mensal inferior a $1 / 4$ do salário mínimo tinham 21 vezes chances a mais de apresentar IA do que aquelas com renda acima de um salário mínimo (SALES-COSTA et al., 2008). Já as famílias com renda mensal inferior a dois salários mínimos apresentavam quase 13 vezes mais chances de estarem em IA moderada e grave e 2,9 vezes mais chances de apresentar IA leve (SALES-COSTA et al., 2008).

A renda escassa pareceu comprometer o pagamento de serviços essenciais, como água, luz e gás e prestações de bens e serviços, além das condições de moradia das famílias em IA (SALES-COSTA et al., 2008; PIMENTEL; SICHIERI; SALLES-COSTA, 2009; PANIGASSI et al., 2008) e, provavelmente, a aquisição de alimentos. A renda insuficiente para a aquisição de alimentos pode refletir negativamente na qualidade da alimentação, com repercussões sobre o estado de saúde dos indivíduos, favorecendo, inclusive, a ocorrência de obesidade e demais doenças crônicas não transmissíveis, que se encontram em ascensão nas famílias pobres brasileiras (OECD, 2018; BORGES et al., 2015; CLARO et al., 2007).

As escolhas alimentares derivam de processos complexos e são influenciadas por aspectos biológicos, sociais, culturais, econômicos e ambientais. 0 preço dos alimentos e as preferências alimentares individuais são importantes determinantes da aquisição e do consumo de alimentos (BORGES et al., 2015; CLARO et al., 2007; MONTEIRO et al., 2018; LOUZADA et al., 2015). O preço pode ser afetado pela disponibilidade e tipo de estabelecimentos que comercializam alimentos e também pelo status socioeconômico do território (DURAN et al., 2013), de modo tal que áreas em desvantagem socioeconômica podem enfrentar um duplo risco - o acesso limitado aos alimentos saudáveis e a baixa qualidade dos alimentos disponíveis (DURAN et al., 2013; LOPES et al., 2017). Além disso, 
em um contexto de desigualdades sociais, a tendência é que famílias com menor renda provavelmente também vivam em áreas que oferecem pouca infraestrutura, reduzido acesso a serviços, pior qualidade residencial e maiores riscos ambientais, potencializando as dificuldades de alcançar os direitos humanos à alimentação e à saúde (NEGRI, 2008; LOPES et al., 2017; OECD, 2018).

Este estudo avança ao investigar as condições do ambiente e do domicílio para além da inadequação do saneamento básico, que habitualmente é investigado na literatura como associado à situação de IA (BRASIL, 2015; SALES-COSTA et al., 2008). Dessa forma, foi possível identificar que, entre estas famílias, também não era plenamente atendido o direito à moradia adequada, uma vez que as áreas apresentavam insuficientes limpeza e manutenção das ruas, limitado acesso a espaços de esporte e lazer, presença de violência ou vandalismo, com inadequados serviços de transporte coletivo, saúde e educação (BRASIL, 2013). Tais resultados reafirmam as desigualdades sociais vividas no país, que comprometem a saúde e qualidade de vida das famílias brasileiras.

A desigualdade de oportunidades de educação, trabalho, atividade física, mobilidade, lazer, disponibilidade de lojas e acesso a alimentos com qualidade e preço acessível (LOPES et al., 2017; CAIAFFA et al., 2008; MENEZES et al., 2017) se reproduz e perpetua em diferentes domínios da vida, aumentando a vulnerabilidade individual e comunitária e expondo a população a múltiplos fatores de risco sociais e ambientais. Assim, a precariedade e a desigualdade das condições de vida das famílias brasileiras em situação de IA, segundo os dados da POF, sinalizam a necessidade de políticas públicas intersetoriais que abarquem os múltiplos componentes envolvidos na SAN.

Esse estudo apresenta pontos fortes. Primeiro, abrange amostra representativa do Brasil, o que favorece o delineamento de ações focadas nas necessidades da população. Segundo, estima a IA nas famílias brasileiras e a sua associação com piores condições de vida, mesmo tendo o país saído da linha da miséria e do mapa da fome das Nações Unidas, alcançando relevantes avanços sociais. Assim, aponta-se a importância da continuidade da realização da POF para o monitoramento da estimativa de IA, permitindo estudos que descrevam sua evolução, sobretudo no contexto de crise econômica, política e social, como vivenciado nos últimos anos.

Quanto às limitações deste estudo, pondera-se que a categorização da variável desfecho foi realizada por questão simplificada contida na POF, que consiste em apenas uma estimativa da IA. Ademais, esta estimativa permite a classificação dos domicílios em situação de SA ou IA, sem identificar graus de insegurança (leve, moderada ou grave) e, portanto, sem diferenciar a preocupação ao acesso adequado de alimentos do comprometimento na qualidade e quantidade da alimentação e da situação de fome em si (BRASIL, 2015; PÉREZ-ESCAMILLA et al., 2004). Porém, a utilização de dados nacionais com um número expressivo de famílias possibilitou obter resultados relevantes sobre a situação do país, apontando a necessidade de intervenções e políticas públicas visando conter o avanço da IA.

Além disso, a POF 2008/2009 realizou a seleção amostral a partir de cadastros de endereços dos domicílios, não incluindo aqueles que não possuem endereços cadastrados, 
como moradores de áreas subnormais, de assentamentos da reforma agrária e comunidades quilombolas, indígenas e ribeirinhos. Este aspecto pode ter subestimado o número de domicílios em situação de IA e as condições de vida vivenciadas, indicando que a situação pode ser ainda mais grave no país.

Outro ponto importante a ser destacado é a temporalidade da pesquisa, realizada há mais de dez anos. A prevalência de IA, mesmo aferida pela POF, pode ter mudado neste período, principalmente, devido à crise econômica de 2015-2018. No Brasil, a partir de 2005, houve um forte investimento em políticas sociais e crescimento econômico, com redução das taxas de desemprego e reestruturação do mercado de trabalho e consequente melhoria da situação de IA. Entretanto, em 2008, uma crise econômica de dimensão global afetou o país, sendo agravada pela crise política e econômica vivenciada em 2015 , que aumentou o desemprego e reduziu as taxas de crescimento do país. Nesse cenário, políticas de austeridade econômica, como a Emenda Constitucional de teto dos gastos públicos, foram implementadas (POCHMANN, 2015). Todo este contexto pode ter mudado drasticamente para pior os níveis de IA da população brasileira, revelando prevalência ainda superior à encontrada neste estudo.

A restrição de direitos sociais pela crise estrutural do capital, aprofundada a partir de 2008 e amparada pela Proposta de Emenda Constitucional (PEC) n. 287, de dezembro de 2016, constitui a mais agressiva proposta de restrições dos direitos sociais da história da seguridade social, no Brasil (SILVA, 2018).

Para modificar essa exposição desigual às condições de vida que afeta a população brasileira em situação de IA, são necessárias políticas públicas inovadoras e efetivas que contemplem a multidimensionalidade da SAN e busquem remodelar as estruturas de oportunidades socioeconômicas e a garantia do direito humano à alimentação adequada e saudável. Ademais, é necessário empreender esforços para o cumprimento dos objetivos do desenvolvimento sustentável propostos pela Agenda 2030 e assinados pelo Brasil, que, entre outros aspectos, propõem erradicar a pobreza e a fome e promover bem-estar, água potável e saneamento, energia acessível e limpa, urbanização inclusiva e trabalho decente e crescimento econômico para os países (ONU, 2018).

\section{Considerações finais}

Foi elevada a prevalência de IA nas famílias brasileiras, segundo estimativa obtida a partir da POF, sendo fortemente associada às condições de vida. Tais resultados possibilitaram revelar um cenário de grande desigualdade social vivenciado no país, ainda que em um momento favorável de crescimento econômico e de políticas sociais. Entretanto, o momento atual é diverso, com grave crise política e de seguridade social, o que aponta a necessidade de monitoramento contínuo dos níveis de IA, por diferentes métodos e estimativas, visando à repactuação do compromisso do governo com a população na busca pelo direito humano à alimentação para todos os brasileiros. 


\section{Referências}

AIRES, J. S.; MARTINS, M. C.; JOVENTINO, E. S.; XIMENES. L. B. (In)segurança alimentar em famílias de pré-escolares de uma zona rural do Ceará. Acta Paulista de Enfermagem, São Paulo, v. 55, n. 1, p. 102-108, jan. 2012.

ARAÚJO, M. L.; MENDONÇA, R. D.; LOPES FILHO, J. D.; LOPES, A. C. Association between food insecurity and food intake. Nutrition, n. 54, p. 54-59, Oct. 2018.

BORGES, C. A.; CLARO, R. M.; MARTINS, A. P.; VILLAR, B. S. Quanto custa para as famílias de baixa renda obterem uma dieta saudável no Brasil? Cadernos de Saúde Pública, v. 31, n. 1, p. 137-148, 2015.

BRASIL. Ministério do Desenvolvimento Social e Combate à Fome. Instituto Brasileiro de Geografia e Estatística. Ministério do Planejamento, Orçamento e Gestão. Pesquisa Nacional de Amostra de Domicílios: Segurança Alimentar. 2. ed. Rio de Janeiro: IBGE, 2015.

BRASIL. Ministério do Desenvolvimento Social e Combate à Fome. O Estado de Segurança Alimentar e Nutricional no Brasil. Um retrato multidimensional. Relatório 2014. Brasília: Ministério do Desenvolvimento Social e Combate à Fome, 2014.

BRASIL. Secretaria de Direitos Humanos da Presidência da República. Coordenação Geral de Educação em SDH/PR, Direitos Humanos, Secretaria Nacional de Promoção e Defesa dos Direitos Humanos. Direito à moradia adequada. Brasília, 2013.

BRASIL. Ministério da Saúde. Instituto Brasileiro de Geografia e Estatística. Ministério do Planejamento, Orçamento e Gestão. Pesquisa de Orçamentos Familiares 2008-2009: análise do consumo alimentar pessoal no Brasil. Rio de Janeiro: IBGE, 2011.

BRASIL. Conselho Nacional de Segurança Alimentar e Nutricional. Cartilha da Lei de Segurança Alimentar e Nutricional. Brasília: Consea, 2006. Disponivel em: http://www4.planalto.gov.br/ consea/publicacoes/cartilha-losan-portugues. Acesso em: 10 jul. 2018.

CAIAFFA, W. T.; FERREIRA, F. R.; FERREIRA, A. D.; OLIVEIRA, C. L.; CAMARGOS, V. P.; PROIETTI, F. A. Saúde urbana: "a cidade é uma estranha senhora, que hoje sorri e amanhã te devora". Ciência \& Saúde Coletiva, v. 13, n. 6, p. 1785-1796, 2008.

CASTANÕ, L. S. A.; ISAZA, E. J. P. Situación alimentaria y nutricional en Colombia bajo el enfoque de determinantes sociales. Perspectivas en Nutrición Humana, v. 15, n. 2, p. 203-214, 2013.

CLARO, R. M.; CARMO, H. C.; MACHADO, F. M.; MONTEIRO, C. A. Renda, preço dos alimentos e participação de frutas e hortaliças na dieta. Revista de Nutrição, v. 41, n. 4, p. 557-64, 2007.

DURAN, A. C.; DIEZ ROUX, A. V.; LATORRE, M. do R.; JAIME, P. C. Neighborhood socioeconomic characteristics and differences in the availability of healthy food stores and restaurants in Sao Paulo, Brazil. Health \& Place, n. 23, p. 39-47, 2013.

DUTRA, L. V.; FRANCESCHINI, S. C.; MORAIS, D. C.; PRIORE, S. E. Insegurança alimentar e indicadores antropométricos, dietéticos e sociais em estudos brasileiros: uma revisão sistemática. Ciência \& Saúde Coletiva, v. 19, n. 5, p. 1475-88, 2014.

DUTRA, L. V.; MORAIS, D. C.; SANTOS, R. H. S.; FRANCESCHINI, S. C. C.; PRIORE, S. E. Comparação de diferentes métodos de avaliação de segurança alimentar domiciliar na área rural de uma cidade brasileira. Revista Brasileira de Estudos de População, Belo Horizonte, v. 35, n. 2, p. 1-17, 2018.

FAO - Food and Agriculture Organization of the United Nations. The State of Food Security and Nutrition in the World. Building resilience for peace and food security. Rome: FAO, 2017. Disponivel em: https://docs.wixstatic.com/ugd/e62f26_d89fc237802844519cad721333aeee3d. pdf. Acesso em: 12 dez. 2018. 
FAO - Food and Agriculture Organization of the United Nations. The State of Food Insecurity in the World. Meeting the 2015 international hunger targets: taking stock of uneven progress. Rome: FAO, 2015. Disponivel em: http://www.fao.org/3/a-i4646e.pdf. Acesso em: 14 jul. 2018.

INTERLENGHI, G. S.; SALLES-COSTA, R. Inverse association between social support and household food insecurity in a metropolitan area of Rio de Janeiro, Brazil. Public Health Nutrition, Wallingford, v. 18, n. 16, p. 2925-2933, set. 2014.

KAC, G. et al. Severe food insecurity is associated with obesity among Brazilian adolescent females. Public Health Nutrition, Wallingford, v. 15, n. 10, p. 1854-60, jan. 2012.

KEPPLE, A. W.; SEGALL-CORRÊA, A. M. Conceituando e medindo segurança alimentar e nutricional. Ciência \& Saúde Coletiva, v. 16, n. 1, p. 187-199, 2011.

LOPES, A. C. S.; MENEZES, M. C.; ARAUJO, M. L. O ambiente alimentar e o acesso a frutas e hortaliças: "uma metrópole em perspectiva”. Saúde \& Sociedade, v. 26, n. 3, p. 764-773, 2017.

LOUZADA, M. L.; MARTINS, A. P.; CANELLA, D. S.; BARALDI, L. G.; LEVY, R. B.; CLARO, R. M.; MOUBARAC, J. C.; CANNON, G.; MONTEIRO, C. A. Alimentos ultraprocessados e perfil nutricional da dieta no Brasil. Revista de Nutrição, n. 10, p. 49-38, jul. 2015.

MENEZES, M. C.; COSTA, B. V.; OLIVEIRA, C. D.; LOPES, A. C. S. Local food environment and fruit and vegetable consumption: an ecological study. Preventive Medicine Report, n. 5, p. 13-20, 2017.

MONTEIRO, C. A.; CANNON, G.; MOUBARAC, J. C.; LEVY, R. B.; LOUZADA, M. L.; JAIME, P. C. The UN decade of nutrition, the NOVA food classification and the trouble with ultra-processing. Public Health Nutrition, v. 21, n. 1, p. 5-17, 2018.

MORAIS, D. C. (In) segurança alimentar e nutricional e relação com indicadores sociais, econômicos e nutricionais de famílias residentes de zona rural. Dissertação (Mestrado em Ciências) - Universidade Federal de Viçosa, Viçosa/MG, 2014.

NEGRI, S. M. Segregação sócio-espacial: alguns conceitos e análises. Coletâneas do Nosso Tempo, v. 8, n. 8, p. 129-153, 2008.

OECD - Organization for Economic Co-operation and Development. Divided cities: understanding intra-urban inequalities. Detroit, 2018. Disponivel em: https://read.oecd-ilibrary.org/urban-ruraland-regional-development/divided-cities. Acesso em: 14 nov. 2018.

ONU - Organização das Nações Unidas Brasil.Transformando nosso mundo: agenda 2030 para o desenvolvimento sustentável. Disponível em: https://nacoesunidas.org/pos2015/agenda2030/. Acesso em: 12 jul. 2018.

PANIGASSI, G.; SEGALL-CORRÊA, A. M.; MARIN-LEÓN, L.; PÉREZ-ESCAMILLA, R.; SAMPAIO, M. F.; MARANHA, L. K. Insegurança alimentar como indicador de iniquidade: análise de inquérito populacional. Cadernos de Saúde Pública, v. 24, n. 10, p. 2376-84, 2008.

PÉREZ-ESCAMILLA, R.; SEGALL-CORRÊA, A. M.; MARANHA, L. K.; SAMPAIO, M. F.; MARIN-LEON, L.; PANIGASSI, G. An adapted version of the US Department of Agriculture Food Insecurity module is a valid tool for assessing household food insecurity in Campinas, Brazil. Journal Nutrition, v. 134, n. 8, p. 1923-8, 2004.

PIMENTEL, P. G.; SICHIERI, R.; SALLES-COSTA, R. Insegurança alimentar, condições socioeconômicas e indicadores antropométricos em crianças da região metropolitana do Rio de Janeiro/Brasil. Revista Brasileira de Estudos de População, Rio de Janeiro, v. 26, n. 2, p. 283-294, jul./dez. 2009.

POCHMANN, M. Ajuste econômico e desemprego recente no Brasil metropolitano. Estudos Avançados, v. 29, n. 85, p 7-19, 2015. 
SALES-COSTA, R.; PEREIRA, R. A.; VASCONCELLOS, M. T.; VEIGA, G. V.; MARINS, V. M.; JARDIM, B. C.; GOMES, F. S.; SICHIERI, R. Associação entre fatores socioeconômicos e insegurança alimentar: estudo de base populacional na Região Metropolitana do Rio de Janeiro, Brasil. Revista de Nutrição, v. 21, supl. 0, p. S99-S109, 2008.

SILVA, M. L. L. Contrarreforma da Previdência Social sob o comando do capital financeiro. Serviço Social \& Sociedade, São Paulo, n. 131, p. 130-154, 2018.

URQUíA-FERNANDES, N. La seguridad alimentaria en México. Salud Pública México, v. 56, supl. 1, p. S92-S98, 2014.

\title{
Sobre as autoras
}

Melissa Luciana de Araújo é nutricionista, doutoranda em Saúde e Nutrição na Escola de Nutrição, Universidade Federal de Ouro Preto. Pesquisadora do Grupo de Estudos em Agricultura Urbana AUÊ! da Universidade Federal de Minas Gerais. Membro do Grupo de Estudos, Pesquisas e Práticas em Ambiente Alimentar e Saúde (GEPPAAS) da Universidade Federal de Minas Gerais.

Diana Rodrigues Nascimento é nutricionista e membro do Grupo de Estudos em Agricultura Urbana AUÊ! da Universidade Federal de Minas Gerais.

Mariana Souza Lopes é nutricionista, doutora em Saúde Pública. Pesquisadora do Grupo de Pesquisa de Intervenções em Nutrição da Universidade Federal de Minas Gerais.

Camila Mendes dos Passos é enfermeira, doutora em Enfermagem. Docente do Departamento de Medicina e Enfermagem da Universidade Federal de Viçosa.

Aline Cristine Souza Lopes é nutricionista, doutora em Saúde Pública. Docente do Departamento de Nutrição, Escola de Enfermagem, Universidade Federal de Minas Gerais. Líder do Grupo de Pesquisa em Intervenções em Nutrição da Universidade Federal de Minas Gerais.

\section{Endereço para correspondência}

\author{
Melissa Luciana de Araújo \\ Universidade Federal de Minas Gerais, Instituto de Geociências, 2ํandar, sala 2051 \\ Av. Antônio Carlos, 6.627, Pampulha \\ 31270-90 - Belo Horizonte-MG, Brasil \\ Diana Rodrigues Nascimento \\ Universidade Federal de Minas Gerais, Instituto de Geociências, 2ํandar, sala 2051 \\ Av. Antônio Carlos, 6.627, Pampulha \\ 31270-90 - Belo Horizonte-MG, Brasil \\ Mariana Souza Lopes \\ Universidade Federal de Minas Gerais \\ Av. Alfredo Balena, 190, sala 316, Santa Efigênia \\ 30130-100, Belo Horizonte-MG, Brasil \\ Camila Mendes dos Passos \\ Universidade Federal de Viçosa \\ Av. Peter Henry Rolfs, $s / n$. \\ 36570-900 - Viçosa-MG, Brasil \\ Aline Cristine Souza Lopes \\ Universidade Federal de Minas Gerais \\ Av. Alfredo Balena, 190, sala 316, Santa Efigênia \\ 30130-100 - Belo Horizonte-MG, Brasil
}




\section{Abstract}

Living conditions of Brazilian families: an estimation of food insecurity

Aim: To research the association between the living conditions of Brazilian families and the Food Insecurity (FI) estimations. Methods: Cross-sectional study conducted with data from the Household Budget Survey (2008-2009) in rural and urban areas. The estimation of Fl rating was obtained by the question: "Which of the following statements best describes the amount of food consumed by your family?", classified as Fl for answers "usually not enough" or "sometimes not enough". Living conditions were analyzed by economic variables, the household and its surroundings. Poisson regression models with robust variance, adjusted, were used. Results: $39.4 \%$ out of 55.406 households evaluated, were classified as FI. In most (93.9\%) difficulty to make ends meet was identified. Prevalence ratio values, adjusted for monthly income per capita, region and area, showed that all economic and unsatisfactory living conditions, as well as home and surrounding problems were significantly associated with Fl. Conclusions: The prevalence of Fl estimates was high, associated with families' unsatisfactory living conditions. Therefore, there is a need to implement effective intersectorial public policies aimed at reducing inequality.

Keywords: Household budget survey. Food insecurity. Social inequity. Living conditions.

\section{Resumen}

Condiciones de vida de las familias brasileñas: estimación de inseguridad alimentaria

Objetivo: Estudiar la asociación entre las condiciones de vida de las familias brasileñas y la estimación de la inseguridad alimentaria (IA). Métodos: Estudio transversal realizado con datos de la Encuesta de Presupuesto Familiar (2008-2009) en áreas rurales y urbanas. La estimación de la calificación de IA se obtuvo de: "De las siguientes declaraciones, ¿cuál describe mejor la cantidad de alimentos consumidos por su familia?", cuando la respuesta era "normalmente no es suficiente" o "a veces no es suficiente". Las condiciones de vida fueron analizadas por variables económicas, el hogar y su entorno. Se construyeron modelos de regresión de Poisson con varianza robusta y ajustada. Resultados: De los 55.406 hogares investigados, 39,4 \% fueron clasificados en IA, y en la mayoría de ellos se identificó como difícil (93,9\%) llegar a fin de mes con los ingresos disponibles. Los valores de la razón de prevalencia, ajustados por el ingreso mensual per cápita, la región y el área, mostraron que todos los aspectos económicos y las condiciones de vida insatisfactorias, así como los problemas del hogar y su entorno estaban significativamente asociados con la IA. Conclusión: La prevalencia de estimación de la IA fue alta, asociada con las condiciones de vida insatisfactorias de las familias. Por lo tanto, es necesaria la implementación de políticas públicas intersectoriales efectivas destinadas a reducir las desigualdades.

Palabras clave: Encuesta de presupuesto familiar. Inseguridad alimentaria. Inequidad social. Condiciones de vida. 